\title{
A Perturbation Method to Analyze the Effect of Cross Section and Angle of Attack for Some Conical Bodies in Hypersonic Flow
}

\author{
N. Talebanfard and A.B. Rahimi*
}

Faculty of Engineering, Ferdowsi University of Mashhad, P.O. Box No. 91775-1111, Mashhad, Iran

\begin{abstract}
An analysis is performed to study the supersonic flow over conical bodies of three different cross sections circular, elliptic and squircle (square with rounded corners) shaped. Perturbation method is applied to find flow variables analytically. In order to find lift and drag forces the pressure force on the body is found, the component along $\mathrm{x}$ is drag and the component along $\mathrm{z}$ is lift. Three equations are obtained for lift to drag ratio of each cross section. The graphs for $\mathrm{L} / \mathrm{D}$ show that for a particular cross section an increase in angle of attack, increases L/D. Comparing L/D in the three mentioned cross sections it is obtained that $\mathrm{L} / \mathrm{D}$ is the greatest in squircle then in ellipse and the least in circle. The results have applications in design of flying objects such as airplanes where many more seats can be arranged in ellipse and or squircle cross section compared to regular circular case.
\end{abstract}

Keywords: Supersonic flow, conical bodies, lift to drag ratio, squircle cross-section, Euler's, equations, Perturbation techniques.

\section{INTRODUCTION}

The flow past conical bodies has been studied for many different cases. A supersonic compressible three dimensional solution is useful in design of supersonic aircrafts, missiles, rockets and etc. Taylor-Maccoll [1] have investigated the steady supersonic flow past a right circular cone at zero angle of attack, they have reduced the governing equations to a single second-order nonlinear differential equation.

Perturbation method is widely applied to studies of flow on conical bodies. Stone [2, 3] applied the power series expansion for a small angle of attack and obtained a solution via perturbation method. Sims [4] performed a numerical integration for Stone's solution. Hypersonic flows over slender pointed nose elliptic cones at zero incidence [5] and [6] is studied. The flow is sought as a small perturbation from some basic circular cone flow which is basically an ellipse. In these studies only pressure has been obtained for the elliptic cone and there is no calculation and comparisons of the lift to drag coefficient with the basic circular cone. In [7] the geometry of the cone cross sections and surface velocities are expanded in Fourier series, using the supersonic linearized conical flow theory, the flow over slender pointed cones are calculated.

The analysis is similar to that of Doty and Rasmussen [8] and Rasmussen [9] for obtaining solutions for flow past circular cones at small angle of attack. The perturbation expansions which are used are not uniformly valid adjacent to body in the thin vortical layer, but it has been shown that pressure and azimuthal velocity components are valid across the vortical layer.

*Address correspondence to this author at the Faculty of Engineering, Ferdowsi University of Mashhad, P.O. Box No. 91775-1111, Mashhad, Iran; Tel/Fax: (98)511-8763304; E-mail: rahimiab@yahoo.com
Calculation and comparison of the ratio of lift to drag for different cross-section situations are non-existing in the literature. In this paper considering the Stone's perturbation expansions and applying them to three conical bodies with different cross sections as circle, ellipse and squircle at small angle of attack, the solution is obtained analytically. The purpose of the present work is to compare the lift to drag ratio for different cross sections. Therefore, for each case the pressure force is determined by integrating pressure around the body for an arbitrary length and then by calculating the dot product of the pressure force in $x$-direction and $z$ direction the drag and lift forces are obtained, respectively. The results will be useful in increasing the lift to drag ratio for aircrafts, satellites, missiles and space vehicles by changing the shape of the cross section or the angle of attack.

\section{PROBLEM FORMULATION}

Consider a supersonic flow over a conical body with arbitrary cross section. Spherical coordinate system is considered for this problem. Due to high Mach numbers, thin boundary layer and decrease of viscous effects, the governing equations become the Euler's equations.

It is assumed that the equation of the body is as follows,

$\theta_{c}=\delta-\varepsilon \cos n \phi+o\left(\varepsilon^{2}\right)$

$\theta_{c}=\delta$ is a cone with circular cross section in spherical coordinate system and semi-vertical angle of $\delta$. The term $-\varepsilon$ $\cos n \varphi$ is added to produce any arbitrary cross section by changing the values of $\varepsilon$ and $n$, in which $\varepsilon$ is a small parameter as a correction factor for cross section to achieve a convex cross section, this parameter is used as the perturbation factor in the expansions for flow variables and $n$ determines the shape of cross section some of the most practical shapes are obtained by $n=1, n=2$ and $n=4$ which represent circle, ellipse and squircle cross sections 


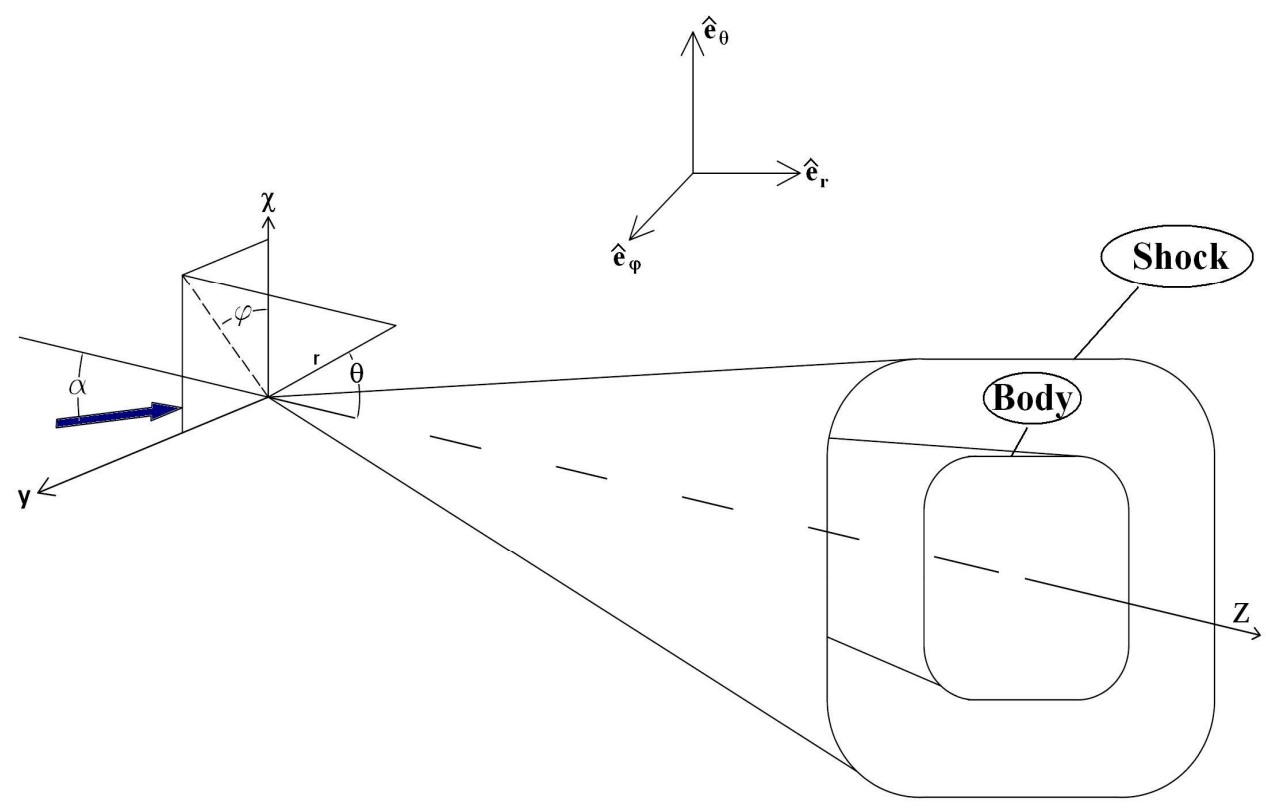

Fig. (1). The geometry and coordinate system.

respectively, hence hereafter these numbers 1,2 and 4 indicate the shape of cross section. Writing the perturbation expansions the following relations are obtained for each flow variable, regarding previous studies in this field W0 is assumed to be negligible [10]. Fig. (1) shows the geometry for the case $n=4$ which is new in the literature.

$$
\begin{aligned}
& u(\theta, \phi, \varepsilon)=u_{0}(\theta)+\varepsilon u_{n}(\theta) \cos n \phi+o\left(\varepsilon^{2}\right) \\
& v(\theta, \phi, \varepsilon)=v_{0}(\theta)+\varepsilon v_{n}(\theta) \cos n \phi+o\left(\varepsilon^{2}\right) \\
& w(\theta, \phi, \bar{\varepsilon})=\bar{\varepsilon} w_{n}(\theta) \sin n \phi+o\left(\bar{\varepsilon}^{2}\right) \\
& p(\theta, \phi, \varepsilon)=p_{0}(\theta)+\varepsilon p_{n}(\theta) \cos n \phi+o\left(\varepsilon^{2}\right) \\
& \rho(\theta, \phi, \varepsilon)=\rho_{0}(\theta)+\varepsilon \rho_{n}(\theta) \cos n \phi+o\left(\varepsilon^{2}\right) \\
& s(\theta, \phi, \varepsilon)=s_{0}(\theta)+\varepsilon s_{n}(\theta) \cos n \phi+o\left(\varepsilon^{2}\right)
\end{aligned}
$$

Substituting the perturbation expansions in the Euler's Equations and separating zero-order and first-order terms in $\varepsilon$, two systems of equations are obtained, since there is no curvature along $r$, derivatives with respect to $r$ are zero, so the systems of equations are simplified. To achieve a complete answer for flow over a conical body at small angle of incidence another perturbation expansion should be written for flow variables in which $\alpha$ (angle of incidence) is the perturbation factor,

$$
\begin{aligned}
& u(\theta, \phi, \alpha)=u_{0}(\theta)+\alpha u_{2}(\theta) \cos \phi+o\left(\alpha^{2}\right) \\
& v(\theta, \phi, \alpha)=v_{0}(\theta)+\alpha v_{2}(\theta) \cos \phi+o\left(\alpha^{2}\right) \\
& w(\theta, \phi, \alpha)=\alpha w_{2}(\theta) \sin \phi+o\left(\alpha^{2}\right) \\
& p(\theta, \phi, \alpha)=p_{0}(\theta)+\alpha p_{2}(\theta) \cos \phi+o\left(\alpha^{2}\right) \\
& p(\theta, \phi, \alpha)=p_{0}(\theta)+\alpha p_{2}(\theta) \cos \phi+o\left(\alpha^{2}\right)
\end{aligned}
$$

$s(\theta, \phi, \alpha)=s_{0}(\theta)+\alpha s_{2}(\theta) \cos \phi+o\left(\alpha^{2}\right)$

in this case the equation of the body is

$\theta_{c}=\delta+\alpha \cos \phi+o\left(\alpha^{2}\right)$

Substituting the perturbation expansions with respect to $\alpha$ in the governing equations, separating zero-order and firstorder terms in $\alpha$, two systems of equations are obtained. Superimposing the solutions for flow variables for each cross section with the solution of flow over a circular cone at small angle of incidence, a complete answer for arbitrary cross section at a small angle of incidence is obtained.

It is obvious that the systems of equations for zero-order in $\alpha$ and $\varepsilon$ are the same (15).

$$
\left\{\begin{array}{l}
2 \rho_{0} u_{0}+\left(\rho_{0} v_{0}\right)^{\prime}+\rho_{0} v_{0} \cot \theta=0 \\
v_{0} u_{0}^{\prime}-v_{0}^{2}=0 \\
\rho_{0} v_{0} v_{0}^{\prime}+\rho_{0} u_{0} v_{0}+\frac{\partial p_{0}}{\partial \theta}=0 \\
v_{0} s_{0}^{\prime}=0 \\
s_{0}=\ln \left(p_{0} M_{\infty}^{2} \gamma\right)-\gamma \ln \rho_{0} \\
\frac{1}{2}\left(u_{0}^{2}+v_{0}^{2}\right)+\frac{\gamma}{\gamma-1} \frac{p_{0}}{\rho_{0}}-\frac{1}{2}-\frac{1}{(\gamma-1) M_{\infty}^{2}}=0
\end{array}\right.
$$

The following system accounts for the first-order terms in $\alpha$ (for $n=1$ ) and $\varepsilon$ (for $n=2,4$ ).

$$
\left\{\begin{array}{l}
2\left(\rho_{0} u_{n}+u_{0} \rho_{n}\right)+\left(\rho_{n} v_{0}+v_{n} \rho_{0}\right)^{\prime}+\cot \theta\left(\rho_{0} v_{n}+v_{0} \rho_{n}\right)+\frac{n \rho_{0} w_{n}}{\sin \theta}=0 \\
v_{n} u_{0}^{\prime}+v_{0} u_{n}^{\prime}-2 v_{0} v_{n}=0 \\
\rho_{0}\left(v_{0} v_{n}\right)+\rho_{n} v_{0} v_{0}^{\prime}+v_{0}\left(\rho_{n} u_{0}+u_{n} \rho_{0}\right)+\rho_{0} u_{0} v_{n}+p_{n}^{\prime}=0 \\
w_{n}^{\prime}+\frac{u_{0}}{v_{0}} w_{n}+w_{n} \cot \theta-\frac{n}{\sin \theta} \frac{p_{n}}{\rho_{0} v_{0}}=0 \\
v_{0} s_{n}^{\prime}+v_{n} s_{0}^{\prime}=0 \\
s_{n}=\frac{p_{n}}{p_{0}}-\gamma \frac{\rho_{n}}{\rho_{0}} \\
\frac{1}{2}\left(u_{0}^{2}+v_{0}^{2}\right)+\left(u_{n} u_{0}+v_{n} v_{0}\right) \frac{\rho_{0}}{\rho_{n}}+\frac{\gamma}{\gamma-1} \frac{p_{n}}{\rho_{n}}-\frac{1}{2}-\frac{1}{(\gamma-1) M_{\infty}^{2}}=0
\end{array}\right.
$$


where $u, v, w$ are velocity components in $r, \theta, \varphi$ directions respectively, $p$ the static pressure, $\rho$ the density of the fluid, $s$ is the entropy and $M_{\infty}$ is the Mach number of the free stream.

Subscript (0) indicates solutions for the basic cone (circular cross section with no angle of attack), subscript $n=$ 1 denotes solutions for circular cone at none zero angle of incidence and for this case $\alpha$ is the perturbation factor, subscripts $n=2,4$, respectivly indicate solutions for ellipse and squircle cross sections and $\varepsilon$ is the perturbation factor.

From the first system the following differential equation with respect to $u_{0}$ is achieved,

$u_{0}^{\prime \prime}+u_{0}^{\prime} \cot \theta+2 u_{0}=0$

The second system of equations leads to the following differential equation with respect to $u_{n}$

$u_{n}^{\prime \prime}+u_{n}^{\prime} \cot \theta+u_{n}\left(2-\frac{n^{2}}{\sin ^{2} \theta}\right)=-\frac{n^{2} F_{n}}{\gamma} \frac{H_{0}(\theta)}{\sin ^{2} \theta}$

The boundary conditions at the body surface are described by the tangency condition.

$v_{0}(\delta)=v_{1}(\delta)=0$

To solve the above differential equations two boundary conditions are required for each equation, from mass conservation across the shock and normal to the shock, the velocity components at the shock are achieved as the following, equations (20) and (21) are the boundary conditions at the shock for equation (17) according to the system of equations (15),

$u_{0}(\beta)=\cos \beta$

$v_{0}^{\prime}(\beta)=-\left(1+\frac{1}{\sigma^{2}}\right)$

$u_{1}(\beta)=\delta \sin \beta\left(1-g_{1}\left(1-\xi_{0}\right)\right)$

$u_{1}^{\prime}(\beta)=-\delta g_{1} v_{0}^{\prime}(\beta)+\delta \xi_{0} \cos \beta\left(1-g_{1}\right)-\xi_{1} \sin \beta$

$\xi_{0}=1-\frac{1}{\sigma^{2}}$

For small angles equation (17) is solved as follows,

$$
\begin{aligned}
& \frac{u_{0}(\theta)}{u_{\infty}}=1-\frac{\theta^{2}}{2}+\left(1-\xi_{0}\right)\left(\beta^{2}\right) \ln \left(\frac{\theta}{\beta}\right) \\
& \frac{v_{0}(\theta)}{u_{\infty}}=-\theta\left[1-\left(1-\xi_{0}\right) \frac{\beta^{2}}{\theta^{2}}\right] \\
& P_{0}(\theta)=\frac{1}{\gamma}\left(\frac{M_{\infty}^{2}}{\exp \left(s_{0}\right)}\right)^{\frac{1}{\gamma-1}}\left[\frac{1}{2}(\gamma-1)\left(1-u_{0}^{2}-v_{0}^{2}\right)+\frac{1}{M_{\infty}^{2}}\right]^{\frac{\gamma}{\gamma-1}} \\
& \rho_{0}(\theta)=\left[\frac{M_{\infty}^{2}}{\exp \left(s_{0}\right)}\left\{\frac{1}{2}(\gamma-1)\left(1-u_{0}^{2}-v_{0}^{2}\right)+\frac{1}{M_{\infty}^{2}}\right\}\right]^{\frac{1}{\gamma-1}}
\end{aligned}
$$

For small angles equation (18) is also solved as follows,

$$
\begin{aligned}
& \overline{u_{1}}(Z)=\frac{u_{1}(z)}{\delta^{2}}=G_{11} Z-G_{12} \frac{1}{Z}+G_{13} R \\
& \overline{v_{1}}(Z)=\frac{v_{1}(Z)}{\delta}=G_{11}+G_{12} \frac{1}{Z^{2}}+G_{13} \frac{d R}{d Z}
\end{aligned}
$$

where,

$$
\begin{aligned}
& G_{11}=\frac{1}{2 \sigma^{2}}+\frac{\gamma-1}{\gamma+1}+g_{1}\left(\frac{2}{\gamma+1}-\frac{1}{2 \sigma^{2}}\right) \\
& G_{12}=\left(\frac{1}{2}-\frac{2 \sigma^{2}}{\gamma+1}\right)+g_{1}\left(\frac{1}{2}+\frac{2 \sigma^{2}}{\gamma+1}\right)+\frac{\left(1-g_{1}\right)}{4 \sigma^{2}} J \\
& G_{13}=\frac{\left(1-g_{1}\right) J}{\sigma^{3}}=\frac{F_{1} J}{N \gamma} \\
& R=1-\frac{3}{4}\left(\frac{\sqrt{z^{2}-1}}{\sqrt{\sigma^{2}-1}}\right)+\frac{2 Z^{2}+1}{4 Z} \frac{\ln (\bar{Z} / \bar{\sigma})}{\sqrt{\sigma^{2}-1}}
\end{aligned}
$$

in which $\bar{\sigma}=\sigma+\sqrt{\sigma^{2}-1}, \bar{Z}=Z+\sqrt{z^{2}-1}$.

$$
\begin{aligned}
& N=\frac{2 \sigma^{2}}{\left(\sigma^{2}-1\right)\left(2 \sigma^{2}+\gamma-1\right)} \\
& J=\frac{2 \sigma^{2}\left[\sigma^{2}-1+(\gamma-1) \ln \sigma\right]}{\left(\sigma^{2}-1\right)\left(2 \sigma^{2}+\gamma-1\right)} \\
& F_{1}=\frac{\gamma\left(1-g_{1}\right) N}{\sigma^{3}} \\
& z=\frac{\theta}{\delta} \\
& \sigma=\frac{\beta}{\delta}
\end{aligned}
$$

By use of the boundary condition

$$
\overline{v_{1}}(1)=0 \text {, }
$$

Shock eccentricity caused by the angle of attack is obtained as the following,

$$
\begin{aligned}
& g_{1}=\frac{2+J+2 \sigma^{2}\left[3-\frac{4\left(\sigma^{2}+1\right)}{\gamma+1}\right]-\left(\frac{J}{\sigma \sqrt{\sigma^{2}-1}}\right) \ln (\bar{\sigma})}{4+J-2\left(\sigma^{2}+1\right)\left[1+\frac{4 \sigma^{2}}{\gamma+1}\right]-\left(\frac{J}{\sigma \sqrt{\sigma^{2}-1}}\right) \ln (\bar{\sigma})} \\
& p_{1}(\theta)=F_{1} p_{0}(\theta)-\rho_{0}(\theta)\left[u_{0}(\theta) u_{1}(\theta)+v_{0}(\theta) v_{1}(\theta)\right]
\end{aligned}
$$

To find lift to drag ratio calculations show that

$$
\begin{aligned}
& p_{0}(\delta)=p_{\infty}\left(1+\frac{\gamma}{2} k_{\delta}^{2}\left(1+\frac{\sigma^{2} \ln \sigma^{2}}{\sigma^{2}-1}\right)\right) \\
& \frac{c_{p_{0}}}{\delta^{2}}=1+\frac{\sigma^{2} \ln \sigma^{2}}{\sigma^{2}-1}
\end{aligned}
$$


$\frac{c_{p_{1}}}{\delta}=\frac{2 N}{k_{\delta}^{2}}\left(1+\frac{\gamma}{2} k_{\delta}^{2}\left(1+\frac{\sigma^{2} \ln \sigma^{2}}{\sigma^{2}-1}\right)\right)\left(\frac{1-g_{1}}{\sigma^{3}}-\frac{a_{0}^{2}(\beta)}{a_{0}^{2}(\delta)} \frac{u_{1}(\delta)}{V_{\infty} \delta}\right)$

$\frac{u_{1}(\delta)}{V_{\infty} \delta}=-2+\frac{\left(1-g_{1}\right)}{\sigma^{3}}\left[\frac{4 \sigma^{2}}{\gamma+1}+\sigma^{3}-\frac{\sigma^{2}}{2}+1-\frac{\ln \left(\sigma+\sqrt{\sigma^{2}-1}\right)}{2 \sqrt{\sigma^{2}-1}}\right]$

$\frac{a_{0}^{2}(\beta)}{a_{0}^{2}(\delta)}=1+\frac{(\gamma-1) \sigma^{2}\left[\ln \sigma^{2}+\frac{1}{\sigma^{2}}-1\right]}{\left(\sigma^{2}-1\right)\left(2 \sigma^{2}+\gamma-1\right)}$

$\frac{c_{p_{0}}}{\delta^{2}}$ and $\frac{c_{p_{1}}}{\delta}$ are shown in Figs. $(2,3)$ as the following,

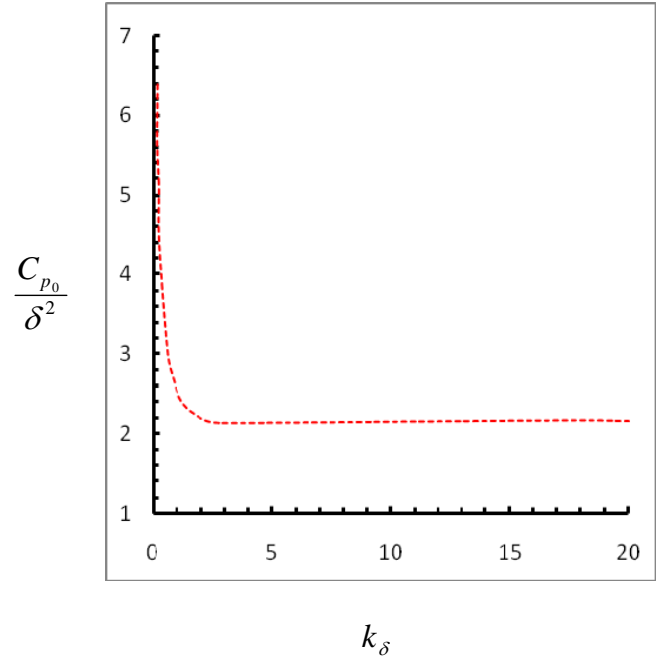

Fig. (2). $c p_{0} / \delta^{2} v s \mathrm{k}_{\delta}$.

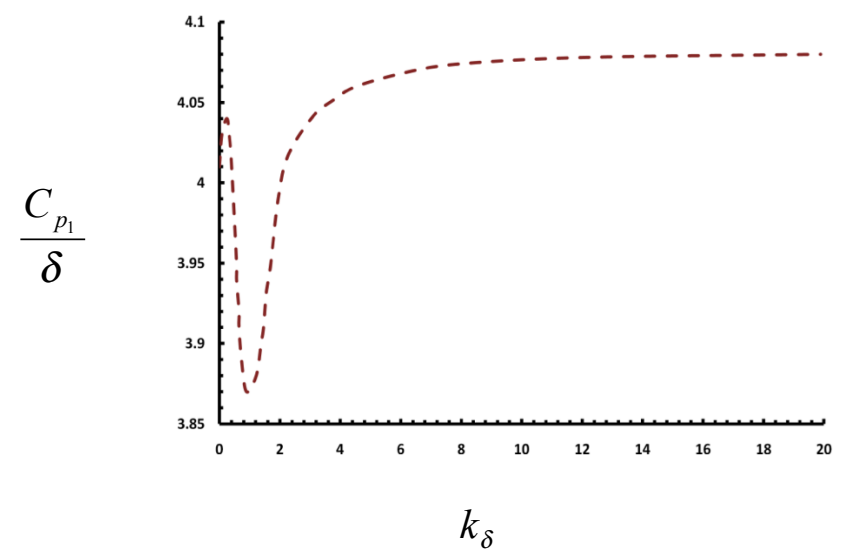

Fig. (3). $c p_{1} / \delta v s k_{\delta}$.

Comparisons of the pressure coefficient from our study with the existing studies are depicted in the two following Fig. $(\mathbf{4}, \mathbf{5})$, which prove well. The numerical results are Sim's results [4] and also a Fluent CFD software has been used by present authors to model the three-dimensional cone. A grid dependence study was conducted to arrive at tetrahedral grid size for optimal accuracy and efficiency for laminar and turbulent cases. For modeling of Reynolds stress in momentum equation, RNG k- $\varepsilon$ turbulence model has been used.

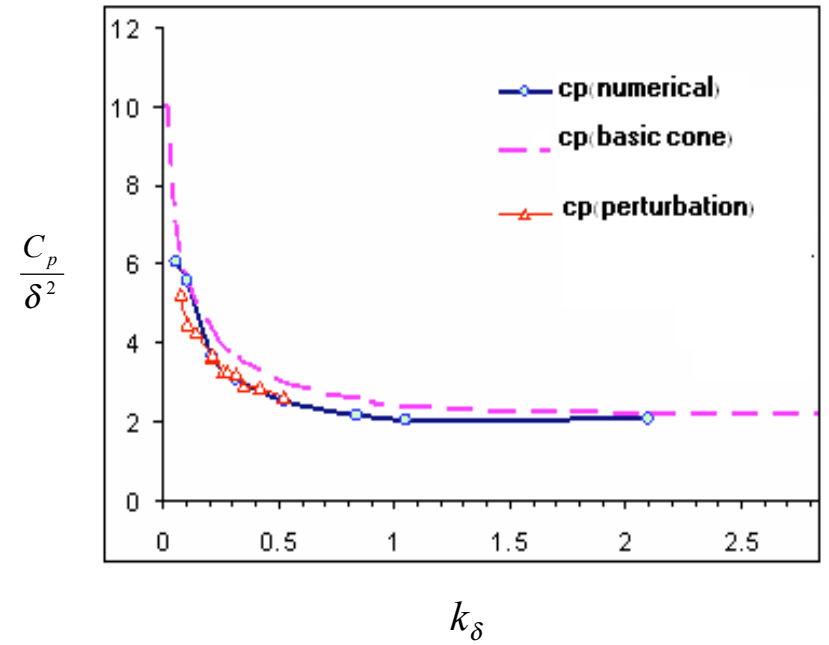

Fig. (4). $C_{p} / \delta^{2} v s k_{\delta}$ in numerical and analytic solution.

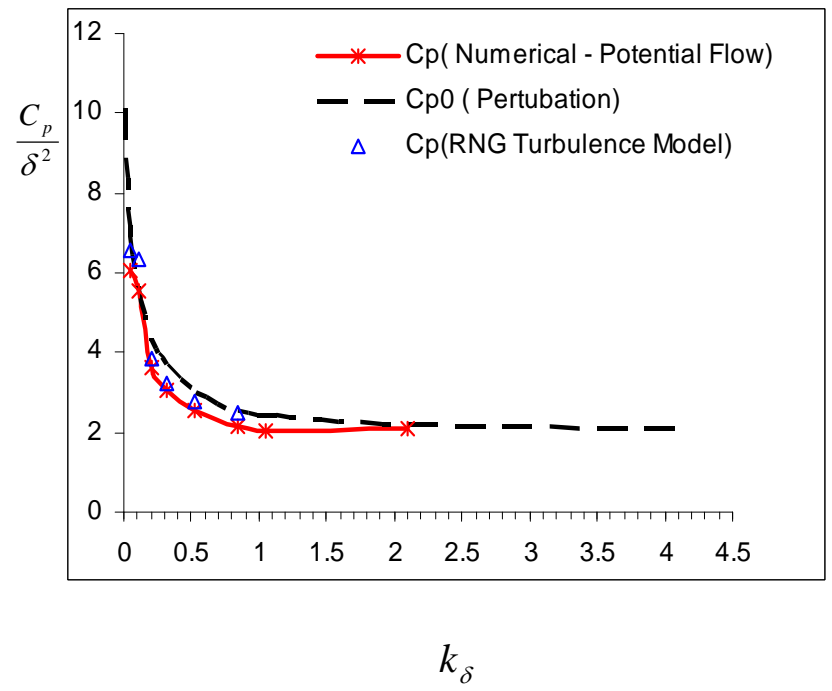

Fig. (5). Analytic solution and numerical solution (basic cone).

\section{CALCULATING LIFT AND DRAG FORCES}

The pressure force on a finite-length cone is given by

$$
\begin{aligned}
& \vec{F}=-\iint_{s} p\left(\theta_{c}\right) \hat{n} d S \\
& \hat{n}=\hat{e}_{\theta}-\frac{n \varepsilon \sin n \phi}{\sin \delta} \hat{e}_{\phi}+o\left(\varepsilon^{2}\right)
\end{aligned}
$$$$
d S=r \sin \theta_{c} d r d \phi+o\left(\varepsilon^{2}\right)
$$

The expressions for Lift and drag forces are as follow,

$$
\begin{aligned}
& D=\vec{F} \cdot \hat{e}_{z}=p_{0}(\delta) \pi H^{2} \tan ^{2} \delta+o\left(\varepsilon^{2}\right) \\
& L=\vec{F} \cdot \hat{e}_{x}=-\frac{1}{4} \frac{c_{p_{1}}}{\delta} \alpha \gamma M_{\infty}^{2} p_{\infty} \pi H^{2} \tan ^{2} \delta+o\left(\alpha^{2}, \varepsilon^{2}, \alpha \varepsilon\right) \\
& \frac{\mathrm{L}}{\mathrm{D}}=-\frac{1}{4 \delta} \frac{c_{p_{1}} \alpha \gamma M_{\infty}^{2} p_{\infty}}{p_{0}(\delta)}
\end{aligned}
$$




\section{RESULTS AND DISCUSSION}

For comparing the lift to drag ratio in different cross sections first the relation between $\delta$ and the shape of cross section should be found.

In rectangle Cartesian coordinates, an elliptic cone is represented by

$$
\frac{x^{2}}{a^{2} z^{2}}+\frac{y^{2}}{b^{2} z^{2}}=1
$$

where Cartesian to spherical transformers are

$$
\begin{aligned}
& x=r \sin \theta \cos \phi \\
& y=r \sin \theta \sin \phi \\
& z=r \cos \theta
\end{aligned}
$$

Substituting (55), (56), (57) in (54) the following relations are obtained.

$$
\tan \theta=\frac{\tan \theta_{m}}{\sqrt{1+e \cos 2 \phi}}
$$

in which

$\tan \theta_{m}=\frac{\sqrt{2} a b}{\sqrt{a^{2}+b^{2}}}=b \sqrt{1-e}$

$e=\frac{b^{2}-a^{2}}{b^{2}+a^{2}}$

In the left hand side of (59) the Taylor expansion about $\varepsilon=0$ is written and in right hand side the Fourier series are substituted. For different values of e calculations shows that Fourier series coefficients except for $\mathrm{a}_{0}$ and $\mathrm{a}_{2}$ are negligible, so the following equation is achieved.

$$
\tan \delta-\varepsilon\left(1+\tan ^{2} \delta\right) \cos 2 \phi=\tan \theta_{m}\left(\frac{a_{0}}{2}+a_{2} \cos 2 \phi\right)
$$

where

$$
\begin{aligned}
& a_{0}=\frac{1}{\pi} \int_{-\pi}^{\pi} \frac{1}{\sqrt{1+e \cos 2 \phi}} d \phi \\
& a_{2}=\frac{1}{\pi} \int_{-\pi}^{\pi} \frac{\cos 2 \phi}{\sqrt{1+e \cos 2 \phi}} d \phi
\end{aligned}
$$

On the other hand $\mathrm{a}_{0} \approx 1$ then for small angles and comparing the two sides of (61) the two following relations are obtained.

$$
\begin{aligned}
& \delta=b \sqrt{1-e} \\
& \varepsilon=-\frac{a_{2} \tan \theta_{m}}{1+b^{2}(1-e)}
\end{aligned}
$$

It is obvious that for a circular cone, $e=0$. $\delta=b$

$\varepsilon=0$

In rectangle Cartesian coordinates, a cone with squircle cross section is represented by

$\frac{x^{4}}{z^{4}}+\frac{y^{4}}{z^{4}}=R^{4}$

The Cartesian to Spherical Transformers are substituted in the Cartesian equation of squircle,

$$
\tan \theta_{c}=\frac{\sqrt{2} R}{(3+\cos 4 \phi)^{1 / 4}}
$$

Using (1) and Taylor Expansion about $\varepsilon=0$ for the left hand side of (69) and writing Fourier series for the right hand side, the following relation is obtained.

$$
\tan \delta-\varepsilon\left(1+\tan ^{2} \delta\right) \cos 4 \phi=\frac{a_{0}}{2}+a_{4} \cos 4 \phi
$$

In comparison with $a_{0}, a_{4}$ the other coefficients of the Fourier Series are much more smaller and hence negligible.

Comparing the two sides of (70) the following relations are achieved.

$$
\begin{aligned}
& \tan \delta=\frac{a_{0}}{2} \\
& \varepsilon=-\frac{a_{4}}{1+\left(\frac{a_{0}}{2}\right)^{2}}
\end{aligned}
$$

in which

$$
\begin{aligned}
& a_{0}=\frac{1}{\pi} \int_{-\pi}^{\pi} \frac{\sqrt{2} R}{(3+\cos 4 \phi)^{1 / 4}} d \phi \\
& a_{4}=\frac{1}{\pi} \int_{-\pi}^{\pi} \cos 4 \phi \frac{\sqrt{2} R}{(3+\cos 4 \phi)^{1 / 4}} d \phi
\end{aligned}
$$

$R$ is a constant and equals to the radius of a circle tangent to the inner side of the squircle.

The lift to drag ratio $v s k_{\delta}$ is shown the following figures, where $k_{\delta}=M_{\infty} \sin \delta$ for small angle of attack $k_{\delta}=M_{\infty} \delta$.

As the angle of attack increases, the lift to drag ratio trends to a constant value for a greater value of $k_{\delta}$ and the value of this constant increases with the increase in angle of attack.

In Figs. $(6,7)$ comparing the lift to drag ratio it is seen that increasing $\mathrm{n}$ from 1 to 4 causes an increase in $\mathrm{L} / \mathrm{D}$, for an ellipse and a squircle which are tangent to the inner side of a circle the lift to drag ratio respectively increases and trends to a constant value which is the greatest for a squircle. 


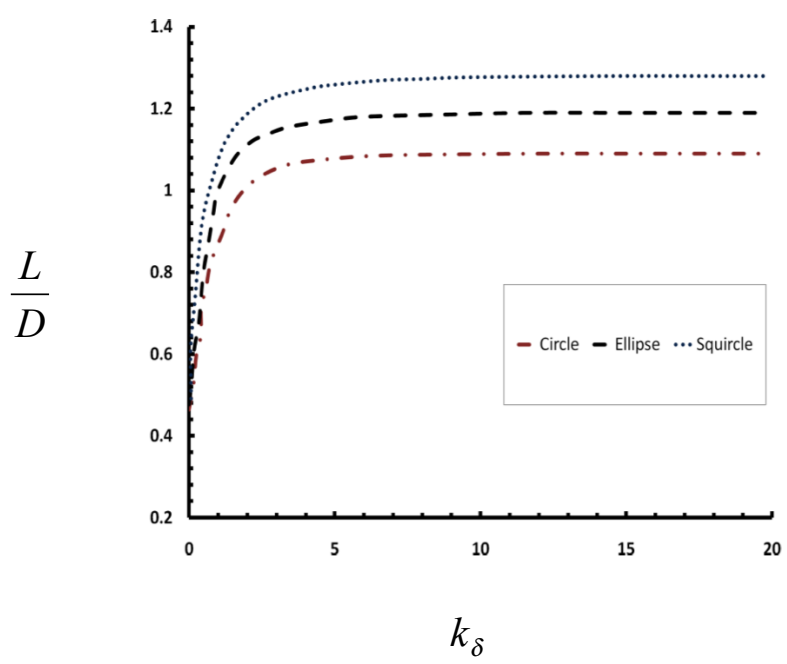

Fig. (6). Lift to drag ratio $v_{s} k_{\delta}, \alpha=4^{\circ}$, semi vertical angle of tangent circular cone is $14.3^{\circ}$.

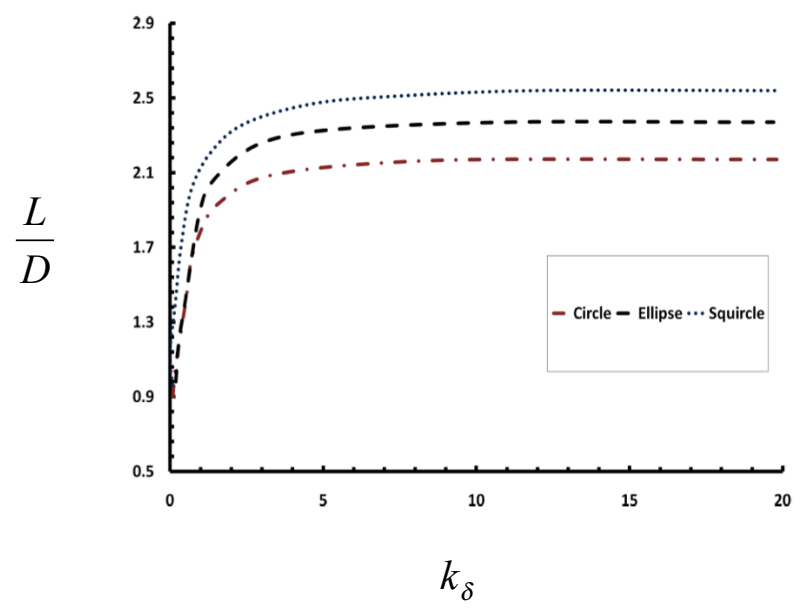

Fig. (7). Lift to drag ratio $v s \mathrm{k}_{\delta}, \alpha=8^{\circ}$, semi vertical angle of tangent circular cone is $14.3^{\circ}$.

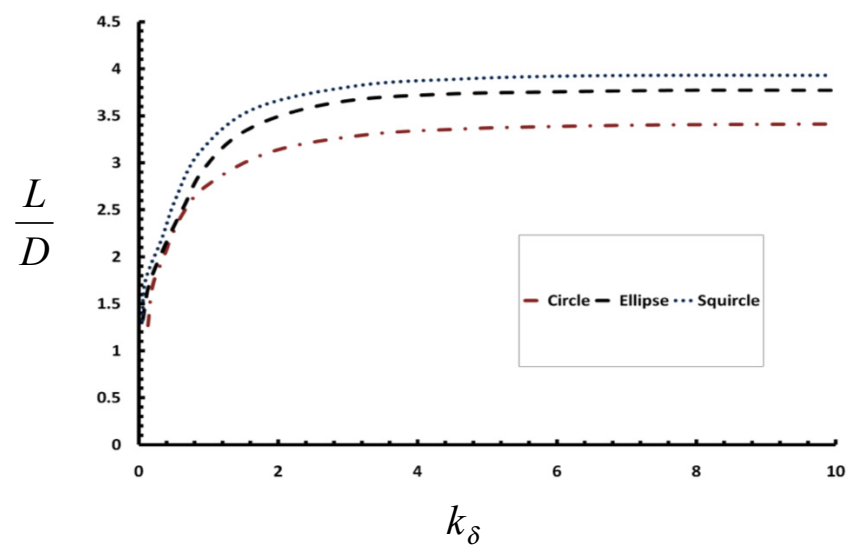

Fig. (8). Lift to drag ration $v s \mathrm{k}_{\delta}, \alpha=8^{\circ}$, semi vertical angle of tangent circular cone is $11.5^{\circ}$.

As expected by decreasing the semi-vertical angle of the cones as shown in Fig. (8) the lift to drag ratio has increases for all of the cross sections because the flow encounters a more slender body. Positioning of cross sections for comparing lift to drag ratio is shown in Fig. (9).
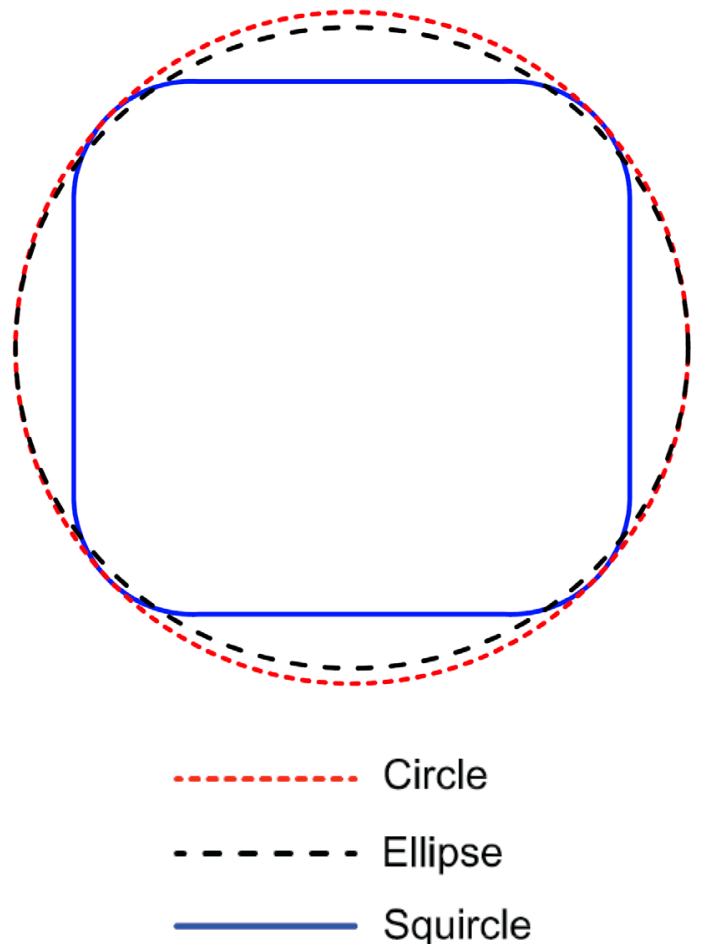

Fig. (9). Positioning of cross sections for comparing lift to drag ratio.

\section{CONCLUSION}

The perturbation method was applied to analytically obtain flow variables over conical bodies of three different cross sections, circle, ellipse and squircle. The aim of the present work is to improve lift to drag ratio by changing the cross section of the conical body. Using Fourier series a relation between $\delta$ and the shape of the cross section of the body is obtained for each case. Theses relations show that by changing the cross section from a circle to an ellipse then to a squircle in a manner that the ellipse and squircle are tangent to the inner side of the circle and the ellipse lies between the other two shapes Figs. $(6,7)$, the lift to drag ratio increases. The results have applications in design of flying objects such as airplanes where many more seats can be arranged in ellipse and or squircle cross section compared to regular circular case.

\section{ACKNOWLEDGEMENT}

This research work has been supported financially by Ferdowsi University of Mashhad based on contract number 3062.

\section{REFERENCES}

[1] G.I. Taylor, and J.N. Maccoll. "The air pressure on cones moving at high speeds", Proc. R. Soc. Lon, Ser. A., vol. 139(A338), pp. 278-311, 1933.

[2] A.H. Stone. On supersonic flow past a slightly yawing cone, $J$. Math. Phys. Vol. 27, pp. 67-81, 1948.

[3] A.H. Stone. on supersonic flow past a slightly yawing cone, $J$. Math. Phys. vol. 30, pp. 220-223, 1952.

[4] J.L. Sims. Tables for supersonic flow around right circular cones at small angle of attack. NASA, SP-3007, 1964.

[5] H.T. Hemdan. "Hypersonic flows over slender pointed-nose elliptic cones at zero incidence" J. Acta Astronaut., vol. 45, no. 1, pp. 1-10, 1999. 
[6] M. C. Jischke. Supersonic flow past conical bodies with nearly circular cross-sections, AIAA-1980-28, $18^{\text {th }}$ Aerospace Sciences Meeting, Jan 14-16, 1980

[7] R. Mascitti. "Calculation of linearized supersonic flow over slender cones of arbitrary cross section", NASA, TN D-6818, 1972.

[8] R.T. Doty, and M.L. Rasmussen. "approximation for hypersonic flow past an inclined cone", J. AIAA, vol. 11, no. 9, 1973.
[9] M.L. Rasmussen, Hypersonic Flow, John Wiley \& Sons, Inc., New York, 1994.

[10] B.J. Tsai and Y.T. Chou. "Analyzing the longitudinal effect of hypersonic flow past a conical cone via the perturbation method", J. Applied Mathematical Modeling, Vol. 32, No. 12 pp. 2596-2620, 2008.

(C) Talebanfard and Rahimi; Licensee Bentham Open.

This is an open access article licensed under the terms of the Creative Commons Attribution Non-Commercial License (http: //creativecommons.org/licenses/by$\mathrm{nc} / 3.0 /$ ) which permits unrestricted, non-commercial use, distribution and reproduction in any medium, provided the work is properly cited. 